\title{
Retrospective analysis of inguinal hernia repair by various methods in a teaching institute
}

\author{
Akruwala SD', Sharma VM² \\ ${ }^{1}$ Dr. Sushil D Akruwala, Assistant Professor, ${ }^{2}$ Dr. Vidhyasagar M Sharma, Assistant Professor. Both from Department of \\ Surgery, GCS Medical College, Hospital \& Research Centre Ahmedabad, Gujarat, India
}

Address for Correspondence: Dr Sushil D Akruwala, Email: drsushilakruwala@gmail.com

\begin{abstract}
Background / Aim: Inguinal hernia is a very common problem. Surgical repair is the current approach. This original article aims to study various methods of inguinal hernia repair over a span of 3 years in a teaching hospital. Methods: All the patients operated electively for uncomplicated inguinal hernia from 2010 to 2012 were selected for the study. They were operated by various methods and followed till 2013. Results: There were total 260 cases of inguinal hernia repair during study period. 210 cases were operated by Lichtenstein method of hernioplasty, 27 by Preperitoneal meshplasty and 23 by TEP. Conclusion: Lichtenstein repair and endoscopic/laparoscopic techniques have similar efficacy. It is found that Lichtenstein's tension free repair is standard and cost effective.
\end{abstract}

Keywords: Inguinal hernia, Meshplasty, Lichtenstein

\section{Introduction}

Approximately $75 \%$ of all abdominal wall hernias are seen in the groin ${ }^{1}$. Inguinal hernia is much more common in men than women. Inguinal hernia repair is one of the most commonly performed surgeries today. Irrespective of country, race or socio-economic status hernia constitutes a major health-care drain.

The aim of this study was to compare the effectiveness and safety of various methods of inguinal hernia repair. The following parameters were evaluated for all different methods of hernia repair.

- Operative technique

- Operating time

- Postoperative pain \& complications

- $\quad$ Long term pain and Recurrence

\section{Materials and Methods}

Patients operated electively for uncomplicated inguinal hernia from 2010 to 2012 were retrospectively studied using a standard form to obtain requisite information. There were total 260 cases of inguinal hernia repair during the said time period and they were followed till 2013.

Manuscript received: $3^{\text {rd }}$ Sept 2013

Reviewed: $26^{\text {th }}$ Sept 2013

Author Corrected: $19^{\text {th }}$ Oct 2013

Accepted for Publication: $20^{\text {th }}$ Nov 2013
All these patients were admitted for planned surgery, they were investigated and preoperative anaesthetic fitness was taken. They were operated as per indication by various methods. Out of all 210 cases were operated by Lichtenstein's repair, 27 by open Preperitoneal meshplasty and 23 by Laparoscopic totally extraperitoneal repair (TEP).

\section{Anaesthetic consideration}

Open mesh repairs were performed under local or locoregional anaesthesia whereas laparoscopic repairs required general anaesthesia.

\section{Method of patient selection}

Lichtenstein's tension free prosthetic repair was standard for us in all unilateral inguinal hernias. Patients with bilateral inguinal hernia were operated by Preperitoneal meshplasty. Those patients who were fit for general anaesthesia and affordable were subjected to laparoscopic totally extraperitoneal repair (TEP). The laparoscopic hernia repair is more difficult in patients who have had previous laparotomy so such patients were operated by Lichtenstein's technique.

Relative contraindications for laparoscopic approach:
A. Obesity with BMI $>30$
B. Significant chest disease
C. Patient on anticoagulants
D. Massive hernias
E. Unfit for GA 


\section{Operating time}

All surgeries were performed by the team led by

1. Seroma / hematoma formation corresponding author. Operative time for each procedure

2. Wound infection was obtained from the records and average was obtained.

3. Postoperative pain (7 days or more)

4. Testicular atrophy

5. Mesh infection

\section{Postoperative complications}

Complications in postoperative period were noted as well as long term sequelae in the form of chronic pain and

6. Chronic pain (more than 6 months)

7. Sinus formation recurrences if any were also recorded.

8. Recurrence

\section{Results}

All patients were male with age ranging from 24 to 78 years with a median of 48.6 years.

Open mesh repairs were performed under local or locoregional anaesthesia. The laparoscopic procedure required general anaesthesia.

\section{Table 1: Type of procedure carried out}

\begin{tabular}{|l|l|}
\hline Type of procedure & Number of patients \\
\hline Lichtenstein method of hernioplasty & $210 / 260(80.76 \%)$ \\
\hline Preperitoneal meshplasty & $27 / 260(10.38 \%)$ \\
\hline TEP & $23 / 260(8.8 \%)$ \\
\hline
\end{tabular}

Out of 260 patients, 212 had unilateral inguinal hernia while 48 had bilateral inguinal hernia. Of these 260 patients 210 were operated by Lichtenstein method, 27 by preperitoneal meshplasty and 23 by TEP.

\section{Table 2: Average time taken for procedure}

\begin{tabular}{|l|l|}
\hline Procedure & Time taken in minutes \\
\hline Lichtenstein method of hernioplasty & 42 minutes \\
\hline Preperitoneal meshplasty & 48 minutes \\
\hline TEP & 65 minutes \\
\hline
\end{tabular}

As per table it is clear that for TEP average time taken was more than other methods. It may be because of more expertise requiring for this procedure.

Table 3: Early complications in different procedures

\begin{tabular}{|l|l|l|l|}
\hline Complications & $\begin{array}{l}\text { Lichtenstein } \\
\text { method(210) }\end{array}$ & $\begin{array}{l}\text { Preperitoneal } \\
\text { meshplasty(27) }\end{array}$ & TEP(23) \\
\hline Seroma/Hematoma & $11(5.2)$ & 0 & $1(4.3)$ \\
\hline Wound infection & $8(3.8)$ & $2(7.4)$ & 0 \\
\hline $\begin{array}{l}\text { Post operative pain } \\
\text { (7days or more) }\end{array}$ & $72(34.2)$ & $3(11.1)$ & 0 \\
\hline Testicular atrophy & 0 & 0 & 0 \\
\hline Mesh infection & 0 & 0 & 0 \\
\hline
\end{tabular}

On comparison of early complication post operative pain was most common $(34.2 \%)$ in Lichtenstein method. Similarly hematoma formation was most common with same technique. 
Table 4: Late complications in different procedures

\begin{tabular}{|l|l|l|l|}
\hline Late complications & Lichtenstein method(210) & $\begin{array}{l}\text { Preperitoneal } \\
\text { meshplasty(27) }\end{array}$ & TEP(23) \\
\hline Chronic pain (6 months or more) & $42(20)$ & $2(7.4)$ & $2(8.6)$ \\
\hline Recurrence & $2(0.9)$ & 0 & 0 \\
\hline Sinus formation & 0 & 0 & 0 \\
\hline
\end{tabular}

Chronic pain was present as late complication in around $20 \%$ of patients with Lichtenstein method. In other techniques chronic pain and other late complication were rare.

All the patients were observed postoperatively. Average length of stay in case of each procedure was recorded. In Lichenstein method it was 4.2 days, preperitoneal method 4.6 days and in TEP 2.8 days.

\section{Discussion}

In our study we included 260 cases of uncomplicated inguinal hernia that presented in our surgical department over the period of three years. All of them were male with median of 48.6 years. Age is a factor for incidence and type of inguinal hernia; incidence increases by age $^{2}$. Inguinal hernia repair is one of the most commonly performed surgery today. All the patients had uncomplicated inguinal hernia. 212 patients had unilateral while 48 had bilateral inguinal hernia.

Numerous repair methods have been described till date. There are three important landmarks in the history of repair of inguinal hernia.

1. Tissue repair (Bassini, Shouldice etc)

2. Tension-free repair (anterior method_Lichtenstein, open posterior method)

3. Laparoscopic hernia repair

Tissue repair methods have now become obsolete and replaced by tension free prosthesis repair. This can be done by anterior approach or posterior approach. Mesh repairs are superior to "nonmesh" tissue-suture repairs.

In laparoscopic repair mesh placement is in preperitoneal plane. The approach may be TAPP (transabdominal preperitoneal) or TEP (totally extraperitoneal). It is associated with longer learning curve and is costlier than open repair.

Patient selection is very important. This needs to take into account patient's fitness for anaesthesia, affordability, history of any previous surgery etc. Locoregional anesthesia is a suitable and economic option for open repairs, and should be popularized in day-care setting. Patients with respiratory and/ cardiovascular diseases are not good candidates for general anaesthesia. Also those patients who had been operated for lower abdominal surgery couldn't be subjected to preperitoneal repair or TEP. Laparoscopic procedure increases cost by use of general anaesthesia and placement of tackers for fixation of mesh.

We at our centre practice Lichtenstein method for unilateral inguinal hernia and Preperitoneal meshplasty for bilateral or recurrent inguinal hernia. We offer TEP laparoscopic procedure to those patients who are fit for general anaesthesia and affordable.

Laparoscopic hernia repair need general anaesthesia, operative time is longer and the risk of serious complications is greater ${ }^{3}$.

In our study the mean operation time for TEP (65 minutes) was slightly longer than Lichtenstein(45 minutes) and preperitoneal method(48 minutes). Compared to other study like Lau $\mathrm{H}$ et $\mathrm{al}^{4}$ where mean time for TEP is $50+/-13.2 \mathrm{~min}$, our time was slightly longer probably due to learning curve.

Among early complications, in Lichtenstein method postoperative pain though mild and easily controlled by single analgesics persisted in 72/210 (34.2\%) patients at the end of 7 days. In the immediate postoperative period we had complications in 19 patients; hematoma and seroma formation requiring drainage, were observed in eight and three patients, respectively. Superficial surgical site infection occurred in 8 patients. It should be emphasized that we have not observed abscess formation or acute infection related to the presence of the foreign body (mesh). Testicular atrophy is an uncommon but well recognised complication of inguinal hernia repair and one that frequently results in litigation ${ }^{5-8}$. None of the patients had testicular atrophy. 
While in preperitoneal meshplasty there was no incidence of seroma/hematoma formation. Two patients had superficial wound infections, postoperatively mild pain persisted at the end of 7 days in 3 patients. Postoperative recovery is short and postoperative pain is minimal ${ }^{9}$.

In TEP, there was hematoma formation in 1 patient which was conservatively managed, but there was no case of wound infection. Pain was minimal in postoperative period and none complained of pain at the end of 7 days. Fewer hematoma/seroma formation were observed in the laparoscopic group in comparison with the Lichtenstein group as in study by Kulacoglu et $\mathrm{al}^{10}$.

Average length of stay was 2.8 days for TEP which was significantly less than Preperitoneal method (4.6days) and Lichtenstein method(4.2days). The reduction in hospital stay after laparoscopic repair is likely to lead to savings in both direct hospital costs and societal costs.

For those surgeons preferring an open approach, the Preperitoneal procedure is a feasible alternative for the standard Lichtenstein procedure and is associated with less chronic pain at six months. Most likely the neuropathic pain and numbness with the Lichtenstein technique are results of more nerves at risk with the anterior approach ${ }^{11}$.

The TEP technique took slightly longer to perform. However it results in very low postoperative pain, fewer wound infection, and quick return to daily activity and working $^{12}$ than patients with Lichenstein method or Preperitoneal method.

Chronic pain has been reported to occur in up to $25-30 \%$ of patients after open inguinal hernia repair ${ }^{13-15}$. In present study chronic pain at the end of 6 months or more was noted in 42/210(20\%) patients with Lichtenstein method and two patients each in TEP and Preperitoneal method. There was no case of delayed mesh infection or sinus formation.

Recurrence rate in our series for Lichtenstein method was comparable with other studies ranging from $0-0.7 \%{ }^{16,17}$. We did not encounter any recurrence in TEP and Preperitoneal meshplasty probably due to limited number of cases.

On the basis of these early experiences, laparoscopic extraperitoneal hernia repair seems to be as good as, if not superior to, the existing open Lichtenstein repair in terms of postoperative pain, hospital stay, return to work, and cosmesis $^{18}$ provided the long-term recurrence rates are also comparable.
However laparoscopic procedure has its own limitations in terms of requirement of general anaesthesia, cost of tackers and learning curve.

Open and laparoscopic/endoscopic techniques have been compared in a number of studies. All laparoscopic repairs are more expensive than open repairs as reported by Hynes et al. in North America ${ }^{19}$, McCormack et al. in the $\mathrm{UK}^{20}$, and Eklund et al in Swedish study ${ }^{21}$. While Lichtenstein method is easy to learn ${ }^{22}$, safe even for beginners and cost effective. At present, the laparoscopic repair of hernias finds its clinical niche in patients with bilateral or recurrent hernias or in patients with unilateral hernia who desire a minimal period of postoperative disability $^{23}$.

\section{Conclusion}

Lichtenstein tension-free mesh inguinal hernia repair is a simple, safe, easy to learn, effective method with low early and late morbidity and remarkably low recurrence rate. Laparoscopic hernia repair is safe and provide less post-operative morbidity and definitely has many advantages over open repair. For bilateral and recurrent inguinal hernias laparoscopic approach is recommended.

\section{Funding: Nil \\ Conflict of interest: Nil \\ Permission from IRB: Yes}

\section{References}

1. Fitzgibbons RJ, Richards AT, Quinn TH. Open hernia repair. In: Souba WS, Mitchell P, Fink MP, Jurkovich GJ, Kaiser LR, Pearce WH, Pemberton JH, Soper NJ, editors. ACS Surgery: Principles and Practice. 6th ed. Philadelphia, U.S.A: Decker Publishing Inc; 2002. pp. 828-849.

2. Ruhl CE, Everhart JE. Risk factors for inguinal hernia among adults in the US population. Am J Epidemiol. 2007;165:1154-1161.

3. George H Sakorafas*, Ioannis Halikias, Christos Nissotakis, NikolaosKotsifopoulos,Alexios Stavrou, Constantinos Antonopoulos and George A Kassaras. Open tension free repair of inguinal hernias; the Lichtenstein technique.BMC Surg. 2001,1:3, doi:10.1186/1471-2482-1-3.

4. Lau H, Patil NG, Yuen WK Day-case endoscopic totally extraperitoneal inguinal hernioplasty versus open Lichtenstein hernioplasty for unilateral primary inguinal hernia in males: a randomized trial. Surg Endosc. 2006 Jan;20(1):76-81. 
5. Reid I, Devlin HB. Testicular atrophy as a consequence of inguinal hernia repair. BJS. 2005;81:91-93.

6. Ibingira CB. Long term complications of inguinal hernia repairs. East Afr Med J. 1999;76(7):396-399.

7. GE Wantz (2000) Testicular atrophy and chronic residual neuralgia as a risk of hernioplasty after inguinal surg. Cl.N. Am 13(3):571-581.

8. Hendry WF. Testicular Epididymal and vassal injuries. BJU Int. 2000;86:344-348.

9. Fenoglio ME, Bermas HR, Haun WE, Moore JT. Inguinal hernia repair: results using an open preperitoneal approach. Hernia. 2005 May;9(2):160-1.

10. Kulacoglu H. Current options in inguinal hernia repair in adult patients. Hippokratia.2001;15(3):223-231.

11. Nienhuijs S, Staal E, Keemers-Gels M, Rosman C, Strobbe L Pain after open preperitoneal repair versus Lichtenstein repair: a randomized trial. World J Surg. 2007 Sep;31(9):1751-7; discussion 1758-9.

12. Karthikesalingam A, Markar SR, Holt PJ, Praseedom RK. Metaanalysis of randomized controlled trials comparing laparoscopic with open mesh repair of recurrent inguinal hernia. Br J Surg.2010;97:4-11.

13. Bay-Nielsen M, Perkins FM, Kehlet H. Pain and functional impairment 1 year after inguinal herniorhaphy: a nationwide questionnaire study. Ann Surg. 2001;233:17.

14. Callesen T, Beck K, Kehlet H. Prospective study of chronic pain after groin hernia repair. Br J Surg.1999; $86: 1528-1531$.

15. Poobalan AS, Bruce J, King PM, Chambers WA, Koukowski ZH, Smith WCS. Chronic pain and quality of life following open inguinal hernia repair. Br J Surg. 2001;88:1122-1126.

16. Kurzer M, Belsham PA, Kark AE. The Lichtenstein repair. Surg Clin North Am. 1998;78:1025-1046.

17. Amid PK, Shulman AG, Lichtenstein IL. Simultaneous repair of bilateral inguinal hernias under local anesthesia. Ann Surg. 1996;223:249-252.

18. Lal P, Kajla RK, Chander J, Saha R, Ramteke VK .Randomized controlled study of laparoscopic total extraperitoneal versus open Lichtenstein inguinal hernia repair. Surg Endosc. 2003 Jun;17(6):850-6.

19. Hynes DM, Stroupe KT, Luo P, Giobbie-Hurder A, Reda D, Kraft M . Cost effectiveness of laparoscopic versus open mesh hernia operation: results of a Department of Veterans Affairs randomized clinical trial. J Am Coll Surg. 2006;203:447-457.

20. McCormack K, Wake B, Perez J, Fraser C, Cook J, McIntosh E, Vale L, Grant A. Laparoscopic surgery for inguinal hernia repair: systematic review of effectiveness and economic evaluation. Health Technol Assess. 2005 Apr;9(14):1-203 iii-iv.

21. Eklund A, Carlsson P, Rosenblad A, Montgomery A, Bergkvist L, Rudberg C. Swedish Multicentre Trial of Inguinal Hernia Repair by Laparoscopy (SMIL) study group. Long-term costminimization analysis comparing laparoscopic with open (Lichtenstein) inguinal hernia repair. Br J Surg.2010;97:765-771.

22. Paajanen H, Varjo R. Ten-year audit of Lichtenstein hernioplasty under local anaesthesia performed by surgical residents. BMC Surg. 2010;10:24.

23. Gainant A. Where does laparoscopy fit in the treatment of inguinal hernia in 2003?. J Chir [Paris]. 2003 Jun; 140 [3]:171-5.

\section{How to cite this article?}

Akruwala SD, Sharma VM. Retrospective analysis of inguinal hernia repair by various methods in a teaching institute. Int $J$ Med Res Rev 2013;1(5):240-244. doi:10.17511/ijmrr.2013.i5.05. 